\title{
Sensory neuropathy in hereditary spastic paraplegia
}

\author{
W Schady, C M L Smith
}

\begin{abstract}
A large kinship is reported with dominantly inherited spastic paraplegia starting in the first decade of life; its clinical evolution was indistinguishable from that of "pure" hereditary spastic paraplegia (HSP). However, all patients studied had electrophysiological evidence of a predominantly sensory polyneuropathy, which was confirmed on nerve biopsy in three. The histological findings indicated virtually complete loss of large diameter fibres with relative preservation of small myelinated and non-myelinated fibres. The neuropathy was largely asymptomatic and there were no trophic ulcers. This family represents a distinct entity which differs from other reported cases of HSP with neuropathy by virtue of the clinical predominance of the pyramidal syndrome, the greater impairment of large fibre sensory modalities than of pain or temperature modalities, and the consequent absence of mutilation.
\end{abstract}

(F Neurol Neurosurg Psychiatry 1994;57:693-698)

The standard form of hereditary spastic paraplegia (HSP), as described by Strümpell, ${ }^{1}$ leads to slowly progressive weakness and spasticity of the legs, sometimes also with increased tendon reflexes in the upper limbs. A number of reports have dealt with complex forms of the disease which may be incorporated under the heading "hereditary spastic paraplegia plus", ${ }^{2}$ and a classification has been attempted by Harding. ${ }^{3}$ Members of such families have been described in whom paraparesis is associated variously with amyotrophy, mental handicap, ichthyosis, macular degeneration, deafness, extrapyramidal features, or cerebellar signs. In many of these kindreds inheritance appears to be recessive, whereas "pure" HSP is usually transmitted as an autosomal dominant trait.

The association of HSP with sensory neuropathy is of particular relevance to this report. Van Epps and Kerr ${ }^{4}$ described four families several members of which had trophic changes in the feet with a background of spastic paraparesis. Painless ulcers developed as a result of profound sensory impairment in the lower limbs. Although the authors considered the disorder to be a form of lumbosacral syringomyelia, Khalifeh and Zellweger ${ }^{5}$ later argued that the lack of dissociation of sensory modalities and the distal symmetrical pattern of sensory loss suggested a neuropathy in association with corticospinal tract disease. Cavanagh et $a l^{6}$ reported five other cases of severe mutilating acropathy in patients with familial spastic paraparesis and provided pathological material in two. In these cases the disease presented in early childhood with developmental delay or recurrent distal painless ulceration. The prognosis was poor, one patient dying at the age of 29 years from secondary amyloidosis and renal failure. The pathological examination in this case and in another patient who had a nerve biopsy showed extensive loss of myelinated and nonmyelinated fibres in peripheral nerves.

Another family is reported with spastic paraplegia and peripheral neuropathy. However, unlike previously reported cases, the clinical picture in this family was virtually indistinguishable from that of "pure" HSP. The sensory neuropathy, although evident on neurophysiological and histological examinations, was largely asymptomatic and there were no trophic ulcers. This indicates that peripheral nerve disease in HSP may be subclinical and go unrecognised unless specifically looked for.

\section{Patients and methods \\ CASE REPORTS}

Case III 5

This 52-year-old man had been unsteady since childhood and was discharged from the army as a result of stiff legs. A physician later described him as having "scissor gait". There was gradual deterioration in his ability to walk and, in his late forties, he began to use a wheelchair. He denied any problems with vision or cutaneous sensation. His mother (Case II 6) had a longstanding disturbance of gait which eventually confined her to a wheelchair. Five of his mother's siblings and his maternal grandmother were similarly afflicted (fig 1). Of his own siblings, five are described later in this report as Cases III 6 to III 10. The youngest brother was examined in another hospital and was found to have a spastic paraparesis dating back to his first decade; two of his three children were affected. Examination in Case III 5 revealed mildly hypertonic arms, severe spasticity of the legs, weakness of hip flexion and foot dorsiflexion, hyperreflexia, ankle clonus, and extensor plantar responses. He could stand and walk short distances with the aid of two sticks. Vibration sense was absent to the level of the hips, and light touch and pinprick sensibility were dulled in his feet. 
Figure 1 Family tree of the reported kinship with hereditary spastic paraplegia. The arrow indicates the proband. Filled symbols represent affected individuals who were examined; partially filled symbols are affected individuals who were not examined.

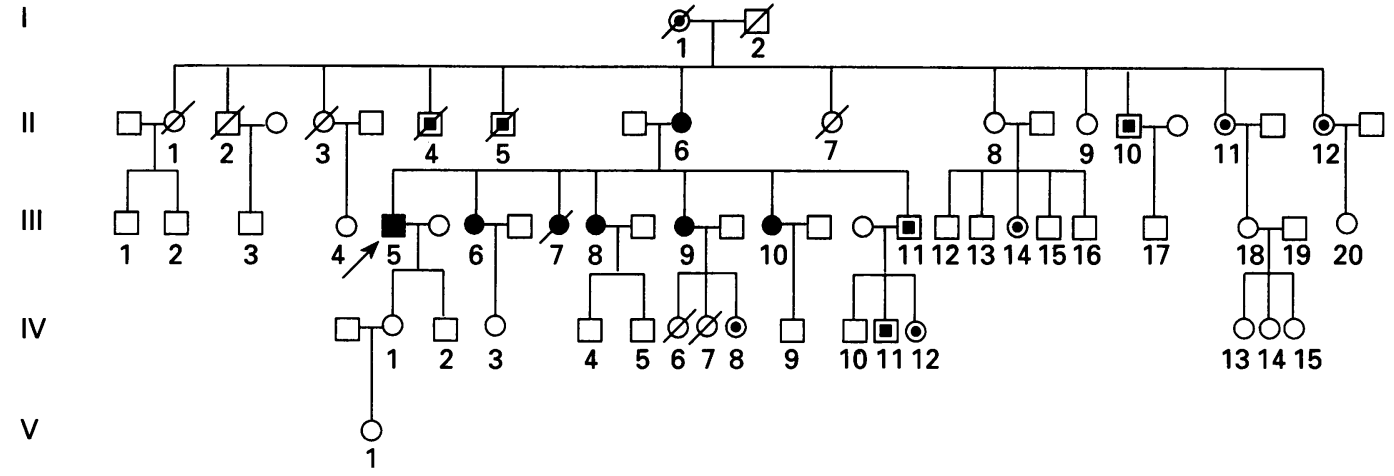

Case III 6

This 51-year-old woman was seen by a paediatrician at the age of 8 years because of difficulty in walking and frequent falls. In her teens she had surgery to lengthen the Achilles tendons. Over the years her lower limbs became stiffer and she developed cramps in her arms. By 1987 she could walk only a few yards unaided; she had a spastic paraparesis with contractures of her calf muscles, and her upper and lower limb reflexes were very brisk. She had clonus at the ankles and bilateral Babinski's signs. Bedside testing of sensation was normal except for mild impairment of joint position sense.

Case III 7

This woman, now 47 years old, started walking on her toes at the age of 11 . After undergoing tenotomies of the Achilles tendon she was fitted with calipers which she has worn ever since. By the fifth decade she needed assistance to walk and she is now virtually wheelchair-bound. On examination, the cranial nerves were normal except for slight nystagmus on right lateral gaze. The upper limbs were mildly spastic and the lower severely so. Hip, knee, and ankle flexors were moderately weak. All tendon reflexes were very brisk and plantar responses were extensor. There was no sensory loss.

\section{Case III 8}

This 44-year-old woman had had stiff legs since childhood. Her symptoms had slowly progressed, especially after two pregnancies in her late thirties. She complained of cold feet but had noticed no numbness. At the time of examination she was able to walk 50 yards with two sticks. She had a severe spastic paraparesis with moderate weakness of lower limb flexor groups, markedly increased knee and ankle jerks, and extensor plantar responses. Perception of pain was reduced in the fingers and feet. Vibration sense was diminished at the ankles and joint position sense was impaired in the toes of both feet, although it was normal in the hands.

\section{Case III 9}

This 40-year-old woman was found to have an atrial septal defect at 18 months. Since the age of 7 years her gait had been awkward, with gradual reduction in exercise tolerance.
She could walk 50-100 yards linked to her husband and used a wheelchair for longer trips. Two of her children were born pre-term and died in infancy; a third had a stiff gait. Examination showed club feet and severely spastic gait. There was mild weakness and wasting of small hand muscles, a moderate paraparesis, increased tone and reflexes in all limbs, and extensor plantar responses. The sense of vibration was lost in the feet and that of joint position sense was impaired.

\section{Case III 10}

This woman, now 41 years of age, had difficulty running at school and had "always" had little control over her left leg. She became weaker with the passage of time, and this was more evident on the left. At the age of 35 she was fitted with orthopaedic shoes. She worked as a kitchen assistant until the age of 37 . When seen in 1987 she could walk 250 yards without aids. Examination revealed normal tone in the limbs, with mild wasting of interossei and moderate asymmetrical weakness of hip flexion and foot dorsiflexion. She had left pes cavus and early contracture of the left calf muscles. All tendon reflexes were very brisk with the exception of an absent right ankle jerk. Plantar responses were weakly extensor. Pinprick sensation was reduced in a glove and stocking distribution. Sense of joint position was impaired in the feet and sense of vibration was absent in the pelvis.

\section{INVESTIGATIONS}

Full blood count, lipid profile, levels of urea, creatinine and hepatic enzymes, cerebrospinal fluid and, in Case III 5, adrenal function tests were normal. Plasma $\beta$-hexosaminidase and other plasma and white cell lysosomal enzymes were present in normal amounts in cases III 5 to III 9. The serum levels of very long chain fatty acids were in the normal range in all patients.

Nerve conduction studies were performed with standard techniques using a Medelec MS6 EMG system (Medelec Ltd, Old Woking, UK). Sensory action potentials (SAPs) were recorded orthodromically in the upper limbs and antidromically in the lower limbs. Stimulation and recording were percutaneous. Compound muscle action potential amplitudes were measured from baseline to negative peak, and those for SAPs from the 
Table Neurophysiological studies in six siblings with hereditary spastic paraplegia

\begin{tabular}{|c|c|c|c|c|c|c|c|}
\hline & \multicolumn{7}{|c|}{ Case No. } \\
\hline & III 5 & III 6 & III 7 & III 8 & III 9 & III 10 & Normal \\
\hline \multicolumn{8}{|l|}{ Median } \\
\hline $\mathrm{MCV}(\mathrm{m} / \mathrm{s})$ & 58 & 60 & 61 & 60 & 55 & 45 & $>46$ \\
\hline \multirow{2}{*}{\multicolumn{8}{|c|}{ Peroneal }} \\
\hline & & & & & & & \\
\hline $\operatorname{MCV}(\mathrm{m} / \mathrm{s})$ & 44 & 46 & 52 & 50 & 43 & 40 & $>40$ \\
\hline CMAP (mV) & 1.5 & $0 \cdot 8$ & $2 \cdot 3$ & $1 \cdot 4$ & $0 \cdot 7$ & 0.6 & $>0 \cdot 8$ \\
\hline \multicolumn{8}{|l|}{ Tibial } \\
\hline $\operatorname{MCV}(\mathrm{m} / \mathrm{s})$ & 43 & - & 35 & 45 & 33 & 42 & $>36$ \\
\hline CMAP (mV) & 0.9 & $1 \cdot 0$ & $3 \cdot 8$ & $1 \cdot 4$ & 0.6 & 0.3 & $>0.8$ \\
\hline Median SAP $(\mu \mathrm{V})$ & 4 & 2 & 5 & 0 & 1 & 0 & $>8$ \\
\hline Ulnar SAP $(\mu \mathrm{V})$ & 1 & 3 & 2 & 0 & 1 & 0 & $>4$ \\
\hline Sural SAP $(\mu \mathrm{V})$ & 0 & 5 & 1 & 0 & 0 & 0 & $>4$ \\
\hline $\operatorname{SEP}(\mu \mathrm{V})$ & $1 \cdot 0$ & $2 \cdot 9$ & $2 \cdot 9$ & - & $1 \cdot 3$ & $1 \cdot 2$ & $>1$ \\
\hline CMCT (ms) & $16 \cdot 1$ & $16 \cdot 2$ & $18 \cdot 1$ & - & 11.9 & - & $<9$ \\
\hline
\end{tabular}

$\mathrm{MCV}=$ motor conduction velocity $\mathrm{CMAP}=$ compound muscle action potential amplitude; $\mathrm{SAP}=$ sensory action potential amplitude; SEP = cortical somatosensory evoked potential amplitude (to median nerve stimulation); CMCT = central motor conduction time (to thenar muscles). Missing values are the result of the patient's intolerance of the procedure.

negative peak to a line drawn from the onset of the response to the positive peak. Results were compared to those obtained from 80 controls of both sexes, aged 19-72 years.

Motor conduction velocities and CMAPs were normal or only slightly reduced (table); SAPs were diminished or absent in all patients. Concentric needle electromyography was abnormal in three of five patients, especially in Case III 9. It showed no spontaneous activity, although motor unit potentials were large (up to $10 \mathrm{mV}$ ) in distal lower limb muscles, suggesting chronic partial denervation and reinnervation. The firing rate was reduced, in keeping with pyramidal tract disease. Cortical somatosensory evoked potential amplitudes were at the lower end of normal. Central motor conduction times to upper and lower limbs, assessed by magnetic stimulation of the motor cortex, were markedly delayed. This finding has been discussed more fully in an earlier paper. ${ }^{7}$

\section{HISTOLOGICAL EXAMINATION OF NERVE \\ Methods}

Whole sural nerve biopsies were obtained from Cases III 5, III 7, and III 10. The nerves were fixed by immersion in 3\% glutaralde- hyde in sodium cacodylate buffer; after fixation they were immersed in $1 \%$ osmic acid and then dehydrated. Lengths of 1-2 mm were embedded in araldite, for both light microscopy, using $1 \mu \mathrm{m}$ sections stained with toluidine blue, and for electron microscopy. Lengths of $1 \mathrm{~cm}$ were placed in non-polymerised araldite for teasing. In addition, part of these $1 \mathrm{~cm}$ lengths was embedded in paraffin, and sections were then stained with haematoxylin and eosin, solochrome cyanin, and Holmes' silver stain for examination by light microscopy. Quantitation was carried out on photomicrographs and electron micrographs, using a Kontron MOP videoplan (Kontron Instruments Ltd, Watford, UK) to calculate the fibre density and produce size-frequency histograms for both myelinated and non-myelinated fibres. These were compared with age and sex matched controls taken from archival postmortem material; this was available in the laboratory from patients who had died from non-neurological disorders.

\section{Results}

The three sural nerves biopsied showed similar pathological features, namely striking loss of myelinated fibres but relative preservation of
Figure 2 Cross-sections (1 $\mathrm{\mu m})$ of sural nerve stained with toluidine blue: $A$ Case III 5, showing a marked reduction in myelinated fibres; $B$ normal control nerve. Magnification $\times 200$, reduced to $66 \%$ for publication)
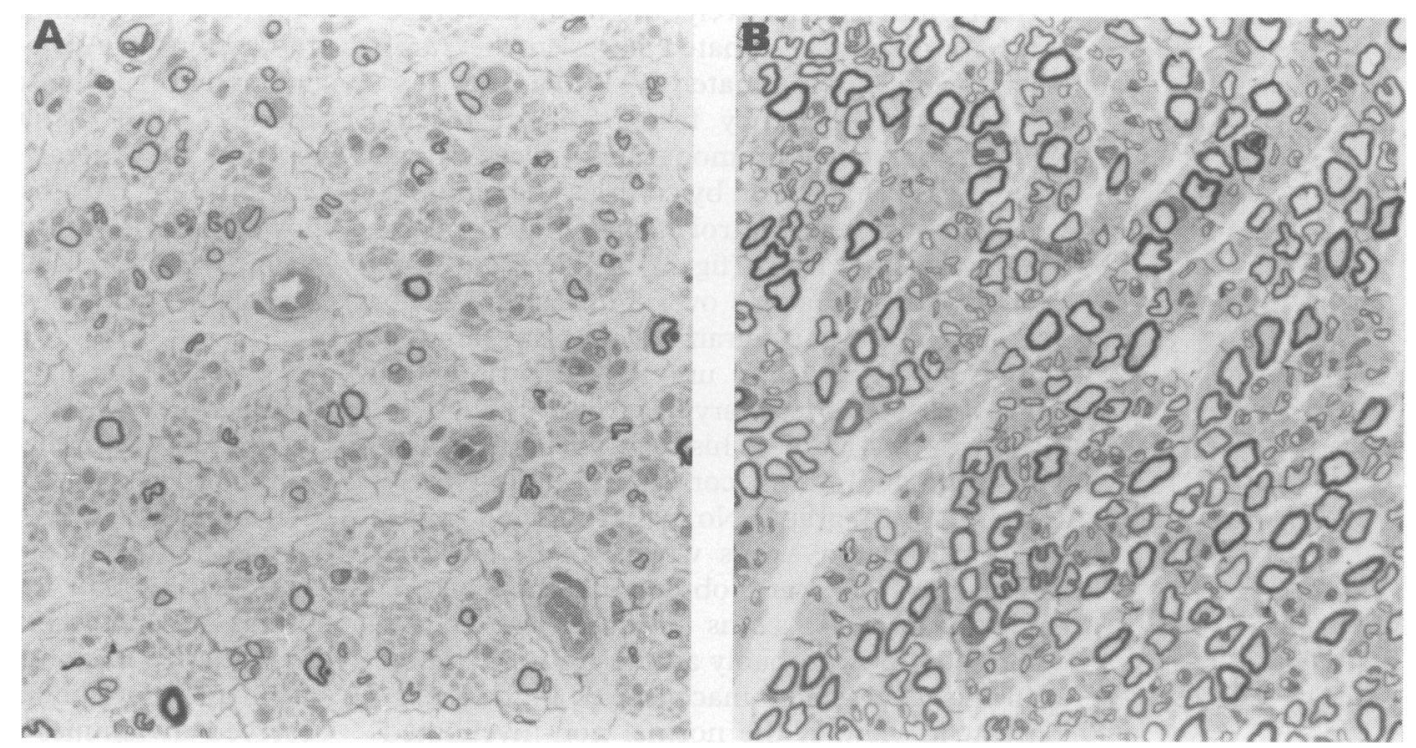

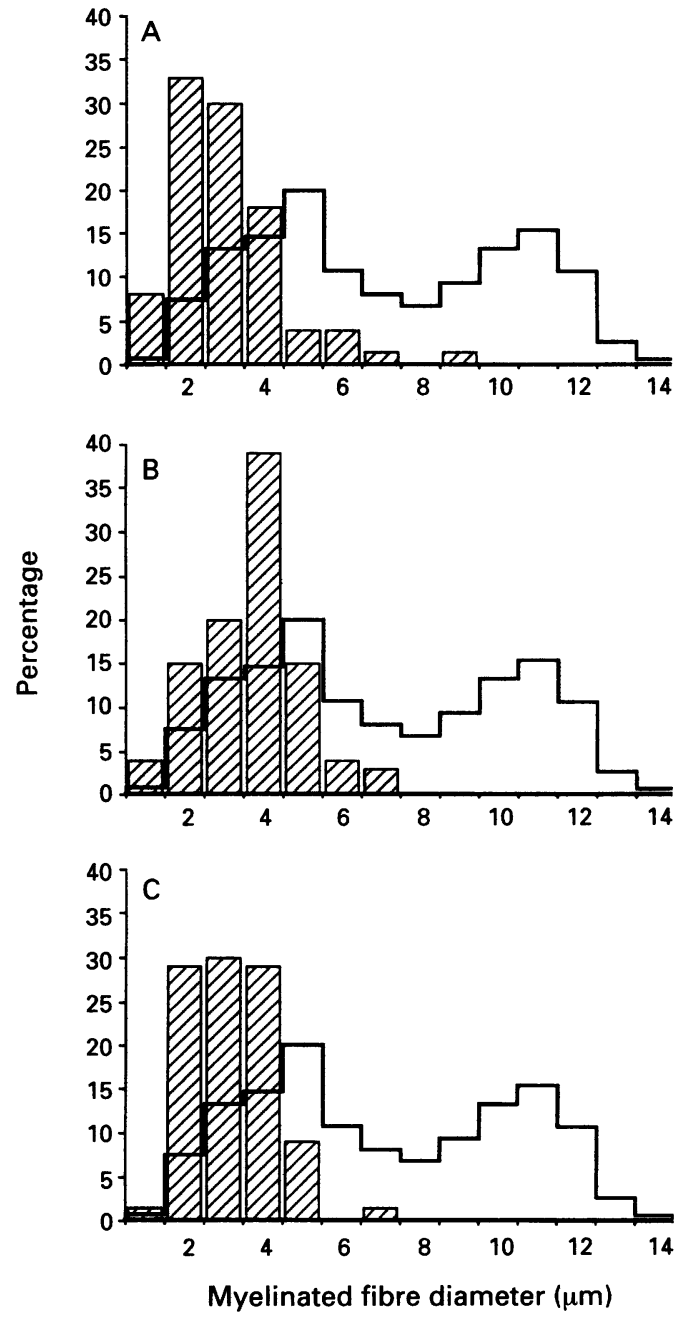

Figure 3 Size-frequency histograms of myelinated fibres in the sural nerves of Cases (A) III 5, (B) III 7, and $(C)$ III 10 (hatched bars), superimposed on the histogram of myelinated fibres in a control nerve (unbroken line).

non-myelinated fibres (fig 2). The average density of myelinated fibres from three fascicles in each of the sural nerves was 2941, 3472 , and $2782 / \mathrm{mm}^{2}$, the normal average density for the sural nerve being $8000 / \mathrm{mm}^{2} .^{8}$ The density of non-myelinated fibres in the three cases was 31778,28888 , and $31111 / \mathrm{mm}^{2}$, respectively, the normal average density of non-myelinated fibres in the sural nerve being approximately $30000 / \mathrm{mm}^{2} .^{8}$

The size-frequency histograms of myelinated fibres were unimodal in all three cases, with peaks at $2-4 \mu \mathrm{m}$; by contrast the bimodal distribution in a control nerve showed peaks at $5 \mu \mathrm{m}$ and $11 \mu \mathrm{m}$ (fig 3). There were very few myelinated axons over $5 \mu \mathrm{m}$ in size. The size-frequency histograms of non-myelinated fibres also showed a unimodal distribution, the size of fibres varying from $0.4 \mu \mathrm{m}$ to $1.8 \mu \mathrm{m}$ (fig 4). These histograms, unlike those of myelinated fibres, correspond quite closely to the control graph. No inappropriately large, non-myelinated axons which might indicate demyelination were observed. The lack of non-myelinated axons in the size range $2 \cdot 0-3 \cdot 0 \mu \mathrm{m}$ is probably a reflection of fixation shrinkage or slight inaccuracies in measurement, in view of the normal non-myelinated fibre density.
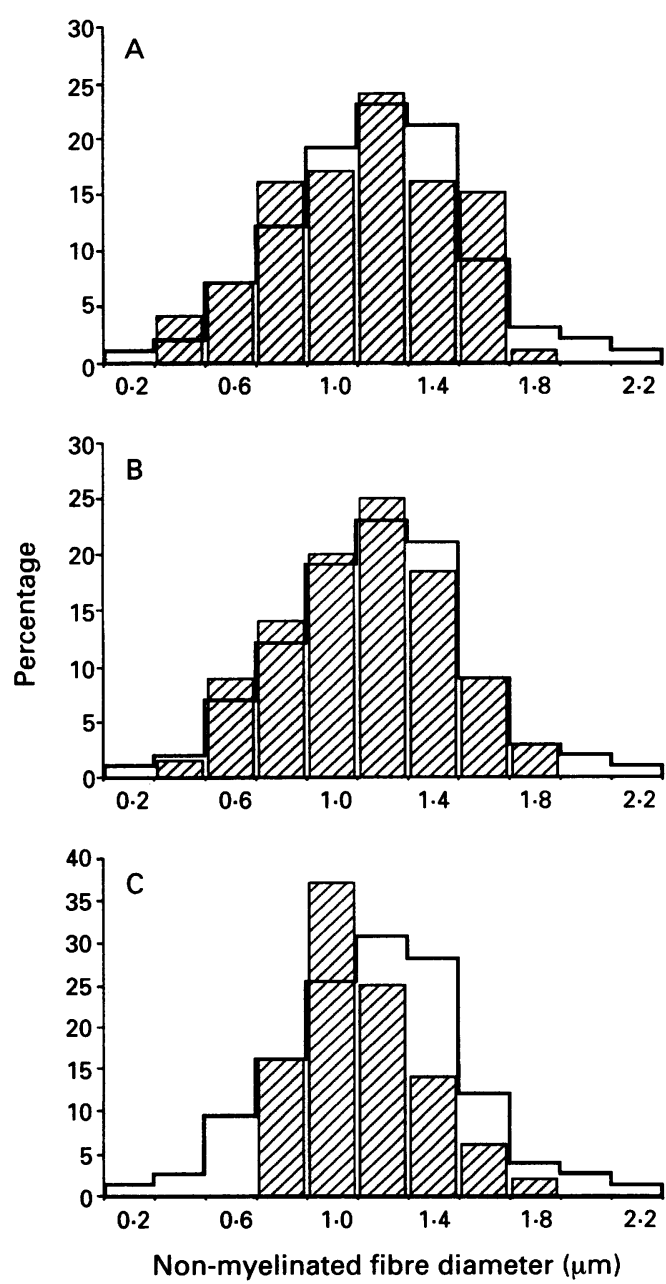

Figure 4 Size-frequency histograms of non-myelinated fibres in the sural nerves of Cases (A) III 5, (B) III 7, and (C) III 10 (hatched bars), superimposed on the histogram of non-myelinated fibres in a control nerve (unbroken line).

On histological examination, the three sural nerves showed an appearance which reflected the quantitative measurements. Occasional clusters of small myelinated axons were seen, which probably represent regenerating fibres. No evidence of active demyelination or remyelination was observed, and this was confirmed by examination of the teased fibre preparations. An occasional fibre was surrounded by a loose whorl of Schwann cell processes, but Schwann cell proliferation was not, in general, a conspicuous feature. The myelin sheaths of myelinated fibres showed normal morphology and periodicity. No inflammatory cell infiltrates or abnormal deposits were noted within the nerve fascicles.

\section{Discussion}

The patients described suffer from autosomal dominant HSP. Members of this kinship have been included in previously reported studies of quantitative sensory assessment and central motor conduction in HSP. ${ }^{79}$ The additional finding of a neuropathy raises the possibility of alternative diagnoses. Combined pyramidal tract and peripheral nerve disease are observed in families with peroneal muscular atrophy with pyramidal features, classified by 
Dyck $^{10}$ as hereditary motor and sensory neuropathy type V. ${ }^{112}$ In these cases, however, the clinical picture is that of distal wasting and weakness, pes cavus, and absent ankle jerks, whereas corticospinal tract dysfunction is less evident. The clinical features of the present patients are more reminiscent of those seen in adrenomyeloneuropathy, ${ }^{13} 14$ although this condition can be excluded by the normal plasma levels of very long chain fatty acids. Hexosaminidase deficiency was ruled out by the normal lysosomal enzyme studies.

There are a number of reports of severe sensory neuropathy in association with HSP..$^{4-6}$ In most of these patients the pyramidal syndrome was overshadowed by chronic painless cutaneous ulcers and neuropathic bone resorption. In other cases trophic skin changes and ulcers in the feet developed on top of a history of established spastic paraparesis. ${ }^{15-17}$ By contrast, the clinical evolution in this present family was identical to that of "pure" HSP. Stiffness of gait developed in the first decade and progressed slowly. There were no complaints of numbness and no amyotrophy or skin ulcers. The examination findings included marked lower limb spasticity, scissoring gait, muscle spasms, ankle clonus, and extensor plantar responses. Cutaneous sensitivity was normal or moderately deranged. Even though there was only sparse clinical evidence of neuropathy, SAPs were reduced or absent, in keeping with loss of afferent axons. Motor conduction was better preserved, although electromyography revealed evidence of partial denervation and reinnervation in three cases.

Sural nerve biopsies in three patients confirmed the axonal nature of the lesion. The size-frequency histograms of myelinated fibres were clearly abnormal-unimodal with peaks at $2-4 \mu \mathrm{m}$. Normal size-frequency histograms for myelinated fibres are bimodal, the external diameter ranging from $2 \mu \mathrm{m}$ to $14 \mu \mathrm{m}$, with peaks for the two populations at $4-5 \mu \mathrm{m}$ and $11 \mu \mathrm{m}$. The pattern of the graphs in the three cases therefore reflects an almost complete loss of myelinated fibres over $4 \mu \mathrm{m}$ in diameter. No inappropriately large myelinated axons were identified and teased nerve fibre preparations showed no evidence of segmental demyelination. Small myelinated fibres were relatively preserved and nonmyelinated axons were present in normal numbers.

The present findings raise the possibility that subclinical neuropathy could be widespread but undetected in HSP, although this is unlikely. In studies of 17 kinships the authors found only two other patients with abnormal SAPs. ${ }^{79}$ McLeod et al. ${ }^{18}$ reported normal median and ulnar SAPs in their 10 patients with HSP. Thomas et al. ${ }^{19}$ found normal median SAPs in all their 18 patients, and Harding $^{20}$ also recorded normal SAPs in the 12 patients tested. It should be borne in mind, however, that in many of these cases sensory nerve conduction studies were limited to the upper limbs, and lower limb abnormalities may have been missed. A case in point is that of Boustany et al., ${ }^{21}$ whose only pointers to a peripheral neuropathy were a delayed tibial $\mathrm{H}$ reflex and diminished sural SAPs.

It is of interest to note that all the present patients had evidence of a sensory polyneuropathy and that the histological abnormalities were similar in the three patients who underwent nerve biopsy, in spite of their age difference. This indicates that neuropathy is an integral part of the syndrome in this particular kinship. Another marker which separates this family is delayed central motor conduction, ${ }^{7}$ making it a distinct syndrome that represents a variant of dominantly inherited HSP. It still needs to be established whether this syndrome has a different genetic and biochemical basis from "standard" HSP. The retained ankle jerks suggest that, in the present patients, muscle afferents were better preserved than cutaneous large myelinated fibres. This contrasts with the early loss of tendon reflexes in Friedreich's ataxia, a condition which, although clinically very different from HSP, is associated with a strikingly similar picture on sural nerve biopsy to that in the present patients. ${ }^{22}{ }^{23} \mathrm{~A}$ large fibre sensory neuronopathy has also been shown in autosomal dominant spinocerebellar degeneration. ${ }^{24}$. Thus there may be a common pattern of afferent neuron atrophy in several of the nervous system inherited degenerations.

1 Strümpell A. Beiträge zur Pathologie des Rückenmarks. Arch Psychiatr Nervenkr 1880;10:676-717.

2 Pratt RTC. The genetics of neurological disorders. Oxford: Oxford University Press, 1967.

3 Harding AE. The hereditary ataxias and related disorders. London: Churchill Livingstone, 1984.

4 van Epps C, Kerr HD. Familial lumbosacral syringomyelia. Radiology 1940;35:160-73.

5 Khalifeh RR, Zellweger H. Hereditary sensory neuropathy with spinal cord disease. Neurology (Minneap) 1963;13:405-11.

6 Cavanagh NPC, Eames RA, Galvin RJ, et al. Hereditary sensory neuropathy with spastic paraplegia. Brain 1979;102:79-94.

7 Schady W, Dick JPR, Sheard A, Crampton S. Central motor conduction studies in hereditary spastic paraplegia. ₹ Neurol Neurosurg Psychiatry 1991;54:775-9.

8 Ochoa J, Mair WGP. The normal sural nerve in man. I. Ultrastructure and numbers of fibres and cells. Acta Neuropathol (Berl) 1969;13:197-216.

9 Schady W, Sheard A. A quantitative study of sensory function in hereditary spastic paraplegia. Brain 1990;113. tion in

10 Dyck PJ. Inherited neuronal degeneration and atrophy affecting peripheral motor, sensory and autonomic neurons. In: Dyck PJ, Thomas PK, Lambert EH, eds Peripheral neuropathy. Philadelphia: WB Saunders, 1975 825-67.

11 Dyck PJ, Lambert EH. Lower motor and primary sensory neuron diseases with peroneal muscular atrophy. I. Neurologic, genetic and electrophysiologic findings in hereditary polyneuropathies. Arch Neurol 1968;18: 603-18.

12 Harding AE, Thomas PK. Peroneal muscular atrophy with pyramidal features. F Neurol Neurosurg Psychiatry 1984; 47:168-72.

13 Griffin JW, Goren E, Schaumburg $\mathrm{H}$, et al. Adrenomyeloneuropathy: A probable variant of adrenomyeloneuropathy: A probable variant of adrenoleukodystrophy. 1. Clinical and endocrinologic

14 O'Neill BP, Marmion LC, Feringa ER. The adrenoleukomyeloneuropathy complex: Expression in adrenoleukomyeloneuropathy complex: E

15 Jequier M, Michail J, Streiff EB. Paraplegie familiale et degenerescence tapetoretinienne. Confinia Neurol 1945;6:277-80

16 Friedman HM, Johnson EW, Liss L. An hereditary radiculospinal degeneration. Neurology (Minneap) 1968;18 308 [abstract].

17 Koenig RH, Spiro AJ. Hereditary spastic paraparesis with sensory neuropathy. Dev Med Child Neurol 1970;12: 576-81. 
18 McLeod JG, Morgan JA, Reye C. Electrophysiological studies in familial spastic paraplegia. $\mathcal{F}$ Neurol Neurosurg Psychiatry 1977;40:611-15.

19 Thomas PK, Jefferys JGR, Smith IS, Loulakakis D. Spinal somatosensory evoked potentials in hereditary spastic paraplegia. F Neurol Neurosurg Psychiatry 1981;44:$$
\text { 243-6. }
$$

20 Harding AE Hereditary "pure" spastic paraplegia: a clinical and genetic study of 22 families. $\mathcal{F}$ Neurol Neurosurg

(1)

21 Boustany R-MN, Fleischnick E, Alper CA, et al. The autosomal dominant form of "pure" familial spastic paraplegia: Clinical findings and linkage analysis of a large pedigree. Neurology 1987;37:910-5.

22 McLeod JG. An electrophysiological and pathologica study of peripheral nerves in Friedreich's ataxia. $\mathcal{F}$ Neurol Sci 1971;12:333-49.

23 Said G, Marion M-H, Selva J, Jamet C. Hypotrophic and dying-back nerve fibers in Friedreich's ataxia. Neurology dying-back nerve fibers in Friedreich's ataxia. Neurology

24 Benett RH, Ludvigson P, DeLeon G, Berry G. Largefiber sensory neuronopathy in autosomal dominant spinocerebellar degeneration. Arch Neurol 1984;41:
175-8. 